\title{
A Broadband Stable Addition Theorem for the Two Dimensional MLFMA
}

\author{
I. Bogaert* and F. Olyslager \\ Ghent University, Department of Information Technology (INTEC), \\ Ghent, Belgium, http://emweb.intec.ugent.be
}

\section{Introduction}

Integral equations arising from the time-harmonic Maxwell equations contain the Green function of the Helmholtz equation as the integration kernel. The structure of this Green function has allowed the development of so-called fast multipole methods (FMMs), i.e. methods for accelerating the matrix-vector products that are required for the iterative solution of integral equations. Arguably the most widely used FMM is the Multilevel Fast Multipole Algorithm (MLFMA). It allows the simulation of electrically large structures that are intractable with direct or iterative solvers without acceleration. The practical importance of the MLFMA is made all the more clear by its implementation in various commercial EM software packages such as FEKO and CST Microwave studio.

However, the MLFMA suffers from the so-called low-frequency (LF) breakdown. This is a numerical instability that prevents the MLFMA from being used at the lower levels of the FMM tree, where sources and observers are less than approximately one wavelength apart. Clearly, this presents a problem when the simulated geometry contains a lot of subwavelength detail. To resolve this problem, the MLFMA is usually paired with another FMM which is then used exclusively for the lower levels. However, such a hybrid scheme has disadvantages too [1]. It would be more elegant, easier to implement and potentially more efficient if one FMM could be made to work for all frequencies, i.e. a broadband FMM. Currently, one such FMM is known, based on the spectral representation of the Green function, though it has the disadvantage of requiring four radiation patterns. In this contribution, a second such FMM will be presented.

More precisely, Gegenbauer's addition theorem in two dimensions will be cast into a form that eliminates the LF breakdown entirely. This full stability means that the new addition theorem is able to achieve machine precision even for the worst possible source-observer configuration occurring in FMMs and for any frequency. This is a clear improvement over previous methods $[2,3]$. In the last section, some numerical results will be shown to showcase the numerical stability of the new addition theorem. However, it also has a clear disadvantage: the number of required discretization points for the addition theorem is far too large to be competitive with other methods. Investigations to solve this problem, for example by means of a QR-decomposition, are in progress. 


\section{A broadband stable addition theorem}

The addition theorem of the MLFMA is given by

$$
H_{0}^{(2)}(k \rho)=\frac{1}{2 \pi} \int_{0}^{2 \pi} e^{-j \boldsymbol{k}(\phi) \cdot \boldsymbol{\rho}_{A}} T\left(k \boldsymbol{\rho}_{T}, \phi\right) \mathrm{d} \phi,
$$

with the wavenumber $k$, the aggregation vector $\boldsymbol{\rho}_{A}=\rho_{A}\left[\cos \phi_{A} \hat{\boldsymbol{x}}+\sin \phi_{A} \hat{\boldsymbol{y}}\right]$, the translation vector $\boldsymbol{\rho}_{T}=\rho_{T}\left[\cos \phi_{T} \hat{\boldsymbol{x}}+\sin \phi_{T} \hat{\boldsymbol{y}}\right]$ and the sum $\boldsymbol{\rho}=\boldsymbol{\rho}_{A}+\boldsymbol{\rho}_{T}$. Also, $H_{n}^{(2)}\left(k \rho_{T}\right)$ denotes the cylindrical Hankel function of the second kind and $n$th order. and $\boldsymbol{k}(\phi)$ is equal to $k[\cos \phi \hat{\boldsymbol{x}}+\sin \phi \hat{\boldsymbol{y}}]$. The so-called translation operator $T\left(k \boldsymbol{\rho}_{T}, \phi\right)$ is given by

$$
T\left(k \boldsymbol{\rho}_{T}, \phi\right)=\sum_{m=-M}^{M} j^{m} H_{m}^{(2)}\left(k \rho_{T}\right) e^{j m\left(\phi-\phi_{T}\right)} .
$$

The addition theorem (1) is numerically unstable at low frequencies because the Hankel function in the translation operator increases super-exponentially if $n>k \rho_{T}$.

To resolve this problem, a different addition theorem will now be constructed, for which the translation operator is inherently stable. We will start by defining the following inner product

$$
\langle f(\phi) \mid g(\phi)\rangle=\frac{1}{2 \pi} \int_{-\infty}^{\infty} \mathcal{F}\left(\phi_{i}\right) \int_{0}^{2 \pi} f\left(\phi_{r}+j \phi_{i}\right) \overline{g\left(\phi_{r}+j \phi_{i}\right)} \mathrm{d} \phi_{r} \mathrm{~d} \phi_{i},
$$

with the function $\mathcal{F}\left(\phi_{i}\right)$ having the property

$$
\int_{-\infty}^{\infty} \mathcal{F}\left(\phi_{i}\right) e^{-2 n \phi_{i}} \mathrm{~d} \phi_{i}=\left|H_{n}^{(2)}\left(k r_{T}\right)\right|^{2}, \forall n \in \mathbb{Z}
$$

It can be proved that such a function can always be found, although it is quite complicated to calculate. When the inner product (3) is applied to ( $2 \pi$-periodic) exponentials, this yields

$$
\left\langle e^{j n \phi} \mid e^{j m \phi}\right\rangle=\delta_{n, m}\left|H_{n}^{(2)}\left(k r_{T}\right)\right|^{2} .
$$

Now define the new, stable, translation operator as follows

$$
T^{\mathrm{new}}\left(k \boldsymbol{\rho}_{T}, \phi\right)=\sum_{m=-M}^{M} j^{-m} \frac{e^{j m\left(\phi-\phi_{T}\right)}}{H_{m}^{(2)}\left(k r_{T}\right)} .
$$

Clearly, this translation operator is very different from (2), mostly because of the division by the Hankel function. The new addition theorem is now constructed as

$$
H_{0}^{(2)}(k \rho)=\left\langle e^{-j \boldsymbol{k}(\phi) \cdot \boldsymbol{\rho}_{A}} \mid T^{\mathrm{new}}\left(k \boldsymbol{\rho}_{T}, \phi\right)\right\rangle .
$$

The validity of (7) can be easily checked. Indeed, since

$$
e^{-j \boldsymbol{k}(\phi) \cdot \boldsymbol{\rho}_{A}}=\sum_{n=-\infty}^{\infty} j^{n} J_{n}\left(k \rho_{A}\right) e^{j n\left(\phi-\phi_{A}\right)}
$$


it follows that

$$
\begin{aligned}
\left\langle e^{-j \boldsymbol{k}(\phi) \cdot \boldsymbol{\rho}_{A}}\right. & \left|T^{\mathrm{new}}\left(k \boldsymbol{\rho}_{T}, \phi\right)\right\rangle \\
& =\sum_{m=-M}^{M} j^{m} \frac{e^{j m \phi_{T}}}{H_{m}^{(1)}\left(\bar{k} r_{T}\right)} \sum_{n=-\infty}^{\infty} j^{n} J_{n}\left(k \rho_{A}\right) e^{-j n \phi_{A}}\left\langle e^{j n \phi} \mid e^{j m \phi}\right\rangle \\
& =\sum_{m=-M}^{M}(-1)^{m} e^{j m\left(\phi_{T}-\phi_{A}\right)} H_{m}^{(2)}\left(k r_{T}\right) J_{n}\left(k \rho_{A}\right)
\end{aligned}
$$

By virtue of the Gegenbauer addition theorem, this yields the required result (7).

The division by the Hankel function in the translation operator (6) plays a key role in attaining numerical stability of (7). The inner product (3) integrates not only over the real part of $\phi$, but also over the complex part. Therefore, the exponential

$e^{j m \phi}$ in the translation operator can become extremely large. The function $\mathcal{F}\left(\phi_{i}\right)$ in the inner product compensates a part of this exponential, but the remaining part is still very large. The Hankel function in the denominator makes sure that this remaining part is reduced to around unity. During the presentation, a proof of the numerical stability will be given.

\section{Numerical results}

The novel addition theorem (7) has been implemented in Matlab and subjected to a practical test. We considered the situation depicted in Fig. 1, which is the typical worst case for FMMs. The points $\boldsymbol{\rho}_{i}$ and $\boldsymbol{\rho}_{j}$ are the source and observer point respectively. $\boldsymbol{\rho}_{l}$ and $\boldsymbol{\rho}_{l^{\prime}}$ are the centers of the two boxes. The target accuracy was then set to $10^{-2}, 10^{-5}, 10^{-8}, 10^{-11}$ and $10^{-14}$. The obtained accuracy for each of these target accuracies is displayed as a function of the wavenumber $k$ in Fig. 2. As can be seen, the novel addition theorem is error-controllable even for the worst case geometry and for very high accuracies.

\section{References}

[1] I. Bogaert, J. Peeters, and F. Olyslager, "A nondirective plane wave MLFMA stable at low frequencies," IEEE Transactions on Antennas and Propagation (in press).

[2] I. Bogaert, D. Pissoort, and F. Olyslager, "A normalized plane wave method for 2-D Helmholtz problems," Microwave and Optical Technology Letters, vol. 48, no. 2, pp. 237-243, February 2006.

[3] I. Bogaert and F. Olyslager, "A low frequency stable formulation of the MLFMA in two dimensions," in Proceedings of the USNC/URSI National Radio Science Meeting, San Diego, USA, 5-12 July 2008. 


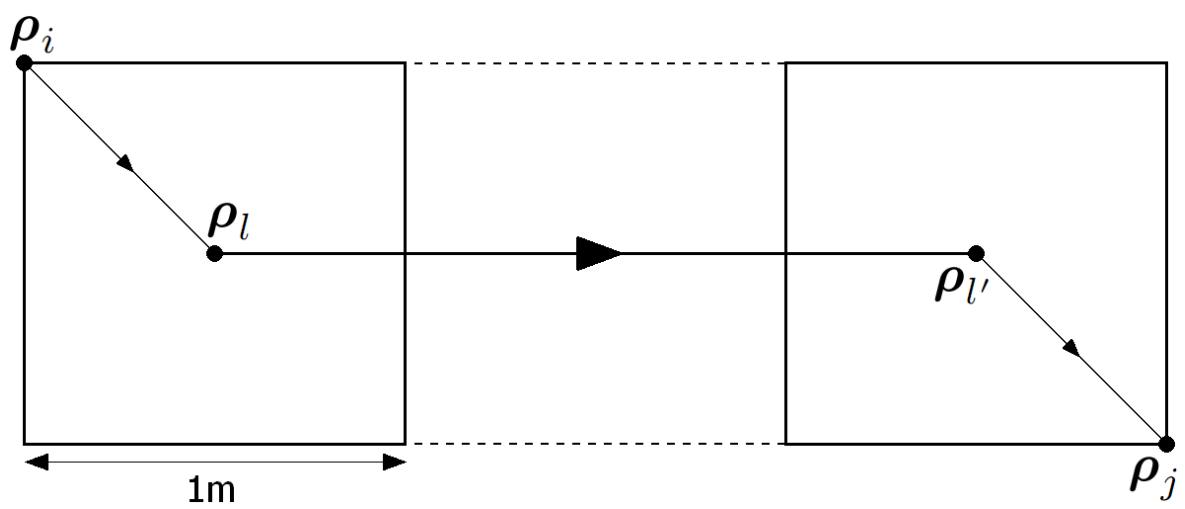

Figure 1: The used geometry for the accuracy test. This is the closest configuration of boxes that occurs in an FMM.

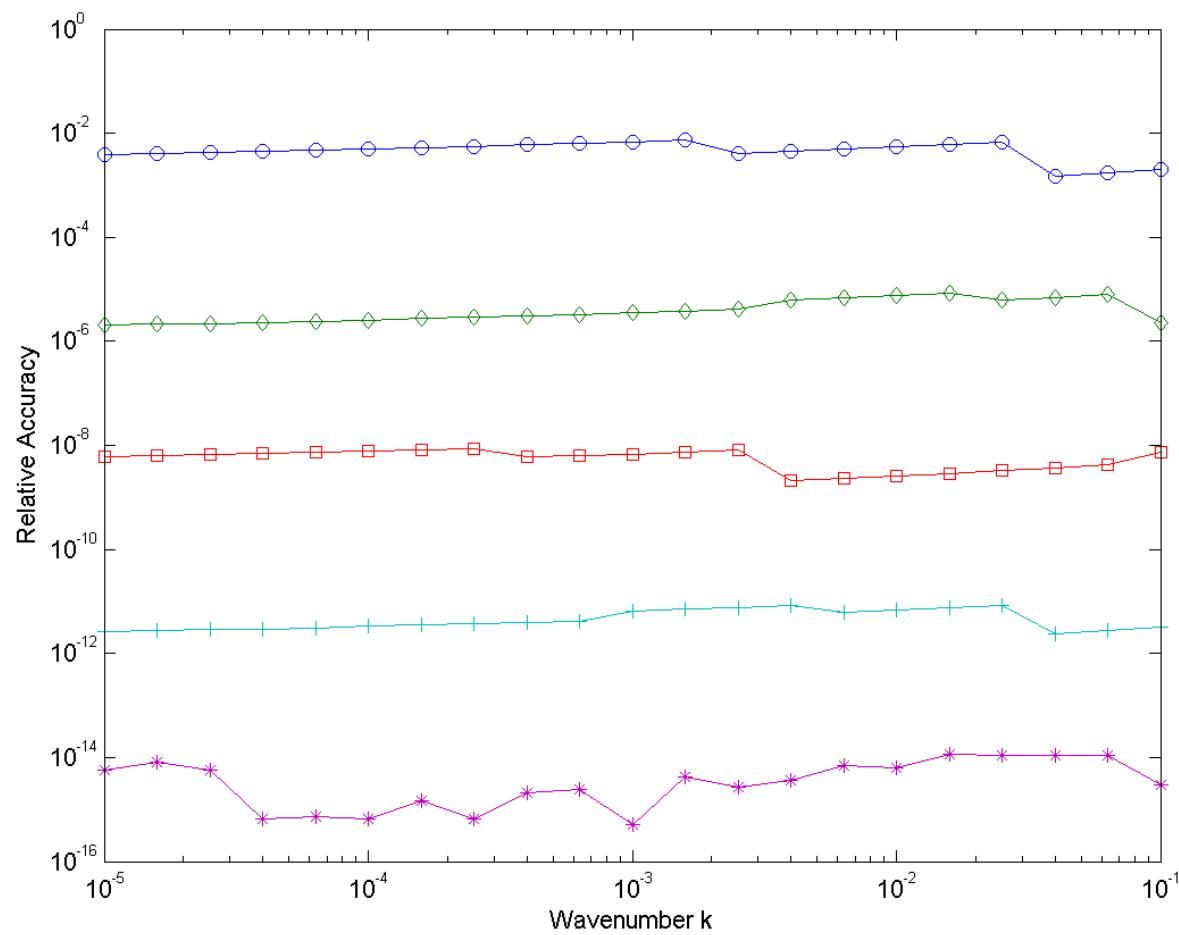

Figure 2: A plot of the relative accuracy between the analytical Green function and the result obtained using the novel addition theorem (7). The used geometry is shown in Fig. 1 and the target accuracies were $10^{-2}, 10^{-5}, 10^{-8}, 10^{-11}$ and $10^{-14}$. Clearly, very high accuracies are possible. 


\section{IEEE International Symposium on Antennas and Propagation and USNC/URSI National Radio Science Meeting}

Copyright and Reprint Permission: Abstracting is permitted with credit to the source. Libraries are permitted to photocopy beyond the limit of U.S. copyright law for private use of patrons those articles in this volume that carry a code at the bottom of the first page, provided the per-copy fee indicated in the code is paid through the Copyright Clearance Center, 222 Rosewood Drive, Danvers, MA 01923. For other copying, reprint, or replication permission, write to IEEE Copyrights Manager, IEEE Operations Center, 445 Hoes Lane, P.O. Box 1331, Piscataway, NJ, 08855-1331. All rights reserved. Copyright (C) 2009 by the Institute of Electrical and Electronics Engineers, Inc. Personal use of this material is permitted. However, permission to reprint/republish this material for advertising or promotional purposes or for creating new collective works for sale or distribution to servers or lists, or to reuse any copyrighted component of this work in other works must be obtained from the IEEE.

IEEE Catalog Number: CFP09APS-CDR

ISBN: 978-1-4244-3647-7

Library of Congress (Serialized): 90-640397

ISSN: 1522-3965

Support: If you have problems or questions related to the installation of this disc, please contact the 3WAIsmen at FAX: (818) 952-0183 or e-mail: wais3men@yahoo.com

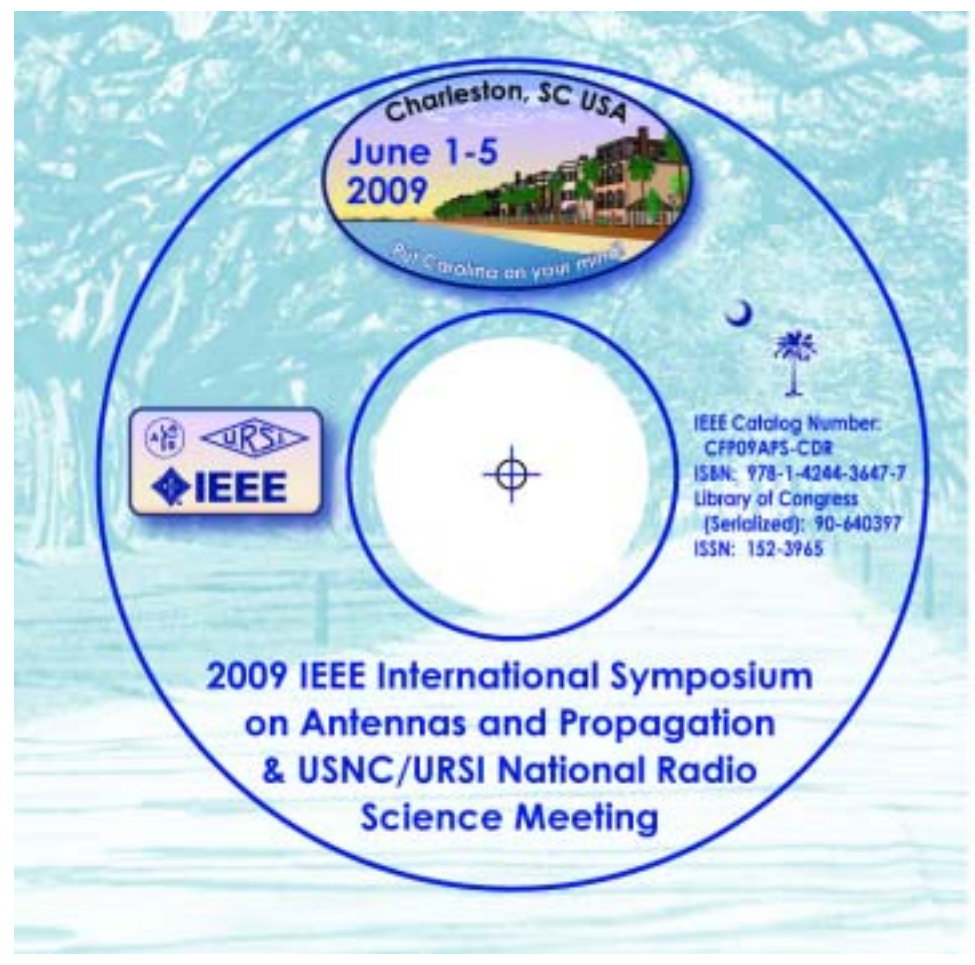

June 1-5, 2009

WA][s\}

Charleston, SC

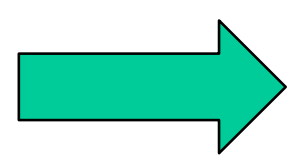

